\title{
On a Model of Leukemia Development with a Spatial Cell Distribution
}

\author{
A. Ducrot ${ }^{a 1}$ and V. Volpert ${ }^{b}$ \\ ${ }^{a}$ UMR CNRS 5466 MAB \& INRIA Futurs Anubis, Université Victor Segalen Bordeaux 2 \\ 33076 Bordeaux, France \\ ${ }^{b}$ UMR CNRS 5208, Université Lyon 1, 69622 Villeurbanne, France
}

\begin{abstract}
In this paper we propose a mathematical model to describe the evolution of leukemia in the bone marrow. The model is based on a reaction-diffusion system of equations in a porous medium. We show the existence of two stationary solutions, one of them corresponds to the normal case and another one to the pathological case. The leukemic state appears as a result of a bifurcation when the normal state loses its stability. The critical conditions of leukemia development are determined by the proliferation rate of leukemic cells and by their capacity to diffuse. The analytical results are confirmed and illustrated by numerical simulations.
\end{abstract}

Key words: leukemia, bone marrow, space cell distribution, reaction-diffusion system, porous medium

AMS subject classification: 92C45, 35K57, 76V05

\section{Introduction}

We study the evolution of leukemia in the bone marrow. Leukemia is a disease arising due to a dysfunction of the hematopoiesis, the process of blood cells production in the bone marrow. It begins with undifferentiated stem cells. They can proliferate or differentiate into more mature cells in order to finally leave the bone marrow to blood vessels. Leukemia changes the process of blood cells production. As a consequence, immature cells or blasts can leave the bone marrow. This is the main observable characteristic of the disease.

\footnotetext{
${ }^{1}$ Corresponding author. E-mail: ducrot@sm.u-bordeaux2.fr
} 
A number of mathematical models has been proposed to understand the hematological cell development and more generally to study cell replication. Most of these models are based on age and maturity structured systems of partial differential equations (see for example $[15,13,14]$ ). They are first applied to study hematopoiesis by M.C. Mackey [6, 7] and further developed in $[8,9,12,1]$. These models lead to delayed partial differential equations. All these studies do not take into account the spatial variables and do not consider the motion of cells in the bone marrow.

Malignant cells can appear in the bone marrow and can proliferate in an abnormal way pushing immature cells out of the bone marrow. Therefore their spatial distribution and their motion can be of primary importance to describe the leukemia development. In this work we describe the proliferation, differentiation and motion of cells in the bone marrow. We also suppose that cells can weakly diffuse in the extra cellular medium.

The paper is organized as follows. Section 2 is devoted to the presentation of the model based on a reaction-diffusion system of equations with convection in a porous medium. Global existence of a one-dimensional solution of the evolution problem is proved in Section 3. Then the existence of a one-dimensional disease free stationary solution is investigated in Section 4 together with its asymptotic stability. When this solution loses its stability, another stationary solution, which corresponds to the leukemic case, bifurcates from it. Finally, Section 5 is devoted to one-dimensional numerical simulations of the model.

\section{The model}

Let us denote by $C_{i}, i=1, \ldots, n$ the concentrations of different cell types in the bone marrow. More precisely, $C_{i}$ denotes the number of cells of the $i^{\text {th }}$ type in a unit volume. Then its evolution is governed by the following equation

$$
\frac{\partial C_{i}}{\partial t}+\nabla \cdot\left(\mathbf{v} C_{i}\right)=d \Delta C_{i}+F_{i}
$$

where $\mathbf{v}$ stands for the velocity, $d$ the diffusion coefficient and $F_{i}$ is the production rate of the $i^{t h}$ type of cells.

We suppose that the bone marrow can be considered as a porous medium and cells as a continuous medium with their velocity described by Darcy's law:

$$
\mathbf{v}=\frac{K}{\mu} \nabla p
$$

Here $p$ is the pressure, $K$ the permeability and $\mu$ the viscosity.

The system (2..1)-(2..2) should be completed by an equation of state. To derive it we assume that all cells have the same volume, they are densely packed and incompressible. This mean that if an additional cell enters a given volume, then another one should leave it. In the other words, we suppose that the total number of cells in the unit volume is constant and denote it by $N$.

Let $\phi$ be the total number of cells in a unit volume, that is

$$
\phi=C_{1}+\ldots+C_{n}
$$


Then we obtain that this quantity is constant. On the other hand, taking a sum of all equations in (2..1), we obtain that $\phi$ satisfies the equation

$$
\frac{\partial \phi}{\partial t}+\nabla \cdot(\mathbf{v} \phi)=d \Delta \phi+\sum_{i=1}^{n} F_{i}
$$

As a consequence, we derive from (2..4) the following equation

$$
\nabla \cdot \mathbf{v}=\frac{1}{N} \sum_{i=1}^{n} F_{i} .
$$

Next, from Darcy's law (2..2) we obtain that the pressure $p$ satisfies the equation:

$$
\Delta p=\frac{\mu}{K N} \sum_{i=1}^{n} F_{i}
$$

We now consider system $(2 . .1),(2 . .2)$ and (2..6) in a two-dimensional rectangular domain

$$
\Omega=\left\{(x, y) \in \mathbb{R}^{2}, \quad 0 \leq x \leq L_{x}, \quad 0 \leq y \leq L_{y}\right\}
$$

The boundary $x=0$ corresponds to the middle axis of the bone marrow. We assume that the solution is symmetric with respect to the middle axis and we consider the right half of the marrow. Therefore, denoting the velocity by $\mathbf{v}=\left(v_{x}, v_{y}\right)$, we have $v_{x}=0$ at $x=0$, so that the pressure satisfies the boundary condition

$$
\frac{\partial p}{\partial x}(0, y)=0 \text { for } y \in\left(0, L_{y}\right)
$$

The right boundary $x=L_{x}$ corresponds to the boundary between the bone marrow and the blood vessel. The boundary condition here is

$$
p\left(L_{x}, y\right)=0 \text { for } y \in\left(0, L_{y}\right) .
$$

Next, we assume that at the top and at the bottom of the domain we have $v_{y}=0$, so that

$$
\frac{\partial p}{\partial y}(x, 0)=\frac{\partial p}{\partial y}\left(x, L_{y}\right)=0 \text { for } x \in\left(0, L_{x}\right)
$$

We discuss now the boundary conditions for the concentrations. For that purpose, we introduce the dimensionless variables

$$
c_{i}=\frac{C_{i}}{N}, \text { for } i=1, . ., n .
$$

We assume that only the cells of the first type can enter the domain, all the other cells can only be produced from the first type of cells inside the domain. These assumptions imply the following boundary conditions

$$
c_{1}(0, y)=1, \quad c_{2}(0, y)=\ldots=c_{n}(0, y)=0 \text { for } y \in\left(0, L_{y}\right) .
$$


We impose the no flux boundary conditions for the concentrations at the top, at the bottom of the domain and at the boundary between the bone marrow and the blood vessel:

$$
\begin{gathered}
\frac{\partial c_{i}}{\partial y}(x, 0)=\frac{\partial c_{i}}{\partial y}\left(x, L_{y}\right)=0 \text { for } x \in\left(0, L_{x}\right) \text { and } i=1, . ., n, \\
\frac{\partial c_{i}}{\partial x}\left(L_{x}, y\right)=0 \text { for } y \in\left(0, L_{y}\right) \text { and } i=1, . ., n .
\end{gathered}
$$

The next step is to specify the production rate, that is the functions $\left(F_{i}\right)_{i=1, . ., n}$. We consider first the normal case and we suppose for simplicity that there are only two types of cells, $A$ and $B$. The first type of cells can proliferate $n$ times giving each time another cell $A$ and a cell $B$. After $n$ divisions the cell dies (or remains alive does not change any more). In order to describe this process we introduce the concentrations $A_{1}, A_{2}, \ldots, A_{n+1}$. Cells of type $A_{1}$ correspond to the original $A$ cells, $A_{2}$ cells correspond to the cells of $A$ type after one division, and so on. We can summarize this construction in the following reaction scheme :

$$
A_{1} \rightarrow A_{2}+B, A_{2} \rightarrow A_{3}+B, \ldots, A_{n} \rightarrow A_{n+1}+B, A_{n+1} \rightarrow P .
$$

Here $P$ denotes the concentration of dead cells. The corresponding kinetics system can be written in the following form

$$
\begin{aligned}
& \frac{d A_{1}}{d t}=-k A_{1} \equiv F_{1}, \\
& \frac{d A_{2}}{d t}=k A_{1}-k A_{2} \equiv F_{2}, \\
& \cdots \\
& \frac{d A_{n}}{d t}=k A_{n-1}-k A_{n} \equiv F_{n}, \\
& \frac{d A_{n+1}}{d t}=k A_{n}-k A_{n+1} \equiv F_{n+1}, \\
& \frac{d B}{d t}=k\left(A_{1}+A_{2}+. .+A_{n}\right) \equiv F_{B}, \\
& \frac{d P}{d t}=k A_{n+1} \equiv F_{P} .
\end{aligned}
$$

We assume for simplicity that the constant $k$ is the same for all cells. Then we obtain

$$
F \equiv F_{1}+\ldots F_{n+1}+F_{B}+F_{P}=k\left(A_{1}+A_{2}+\ldots+A_{n}\right) .
$$

Next, we denote

$$
c_{1}=A_{1}, \ldots, c_{n}=A_{n},
$$

and we deal with the following system

$$
\frac{\partial c_{i}}{\partial t}+\nabla \cdot\left(\mathbf{v} c_{i}\right)=d \Delta c_{i}+\frac{1}{N} F_{i}, \text { for } i=1, \ldots, n,
$$




$$
\mathbf{v}=\frac{K}{\mu} \nabla p, \Delta p=\frac{\mu}{K N} F
$$

together with the corresponding boundary conditions. Here $F$ is defined by the equality

$$
F=k\left(c_{1}+\ldots+c_{n}\right),
$$

while $F_{i}=k c_{i-1}-k c_{i}$ for $i \geq 2$ and $F_{1}=-k c_{1}$. The normal case corresponds to a negligible concentration of immature cells, that is $c_{1}, \ldots, c_{n}$, at $x=L_{x}$.

We now consider the pathological case. We suppose that there is an other type of cells, denoted by $D$, which can proliferate without differentiation and give two daughter cells identical to the mother cell,

$$
D \rightarrow 2 D .
$$

We do not consider mortality of these cells because of a possible dysfunction of the $p 53$ gene and the appearance of immortal cells. The kinetic equation for this reaction scheme has the form:

$$
\frac{d D}{d t}=k_{s} D
$$

Introducing the dimensionless concentration

$$
s=\frac{D}{N}
$$

we have now to deal with system (2..15) together with the equation

$$
\frac{\partial s}{\partial t}+\nabla \cdot(\mathbf{v} s)=d_{s} \Delta s+\frac{k_{s}}{N} s
$$

and the equations of motion:

$$
\mathbf{v}=\frac{K}{\mu} \nabla p, \quad \Delta p=\frac{\mu}{K N}\left(k\left(c_{1}+\ldots+c_{n}\right)+k_{s} s\right) .
$$

The diffusion coefficient $d_{s}$ and the reaction rate $k_{s}$ in equation (2..18) can be different from those in system (2..15). We will see below that the relation between them can be essential for propagation of malignant cells.

In order to study this problem we introduce some notations. We set

$$
\tilde{k}=\frac{k}{N}, \quad \tilde{k}_{s}=\frac{k_{s}}{N}, \quad \nu=\frac{\mu}{K} .
$$

Therefore, omitting the tilde, we obtain the following problem

$$
\begin{gathered}
\frac{\partial c_{i}}{\partial t}+\nabla .\left(c_{i} \mathbf{v}\right)=d \Delta c_{i}+k\left(c_{i-1}-c_{i}\right), \quad i=1, . ., n, \quad c_{0}=0 . \\
\frac{\partial s}{\partial t}+\nabla \cdot(s \mathbf{v})=d_{s} \Delta s+k_{s} s \\
\nabla \cdot \mathbf{v}=k\left(c_{1}+. . c_{n}\right)+k_{s} s, \quad \nabla p=\nu \mathbf{v}
\end{gathered}
$$

together with the corresponding boundary conditions. 


\section{Global existence}

In this section we study the global existence of one-dimensional solutions for the problem:

$$
\begin{gathered}
\frac{\partial c_{i}}{\partial t}+\frac{\partial\left(c_{i} v\right)}{\partial x}=d \frac{\partial^{2} c_{i}}{\partial x^{2}}+k\left(c_{i-1}-c_{i}\right), \quad i=1, \ldots, n, \\
\frac{\partial s}{\partial t}+\frac{\partial(s v)}{\partial x}=d_{s} \frac{\partial^{2} s}{\partial x^{2}}+k_{s} s \\
\frac{\partial v}{\partial x}=k\left(c_{1}+\ldots+c_{n}\right)+k_{s} s
\end{gathered}
$$

$\left(c_{0}=0\right)$ considered in the domain $\Omega=\{x \in \mathbb{R}, \quad 0 \leq x \leq L\}$. The system is completed by the boundary conditions

$$
\begin{aligned}
& c_{1}(0)=1, \quad c_{i}(0)=0 \quad i=2, \ldots, n, \quad s(0)=0, \\
& c_{i}^{\prime}(L)=0, \quad i=1, \ldots, n, \quad s^{\prime}(L)=0, \quad v(0)=0,
\end{aligned}
$$

and initial conditions

$$
c_{i}(0, x)=c_{i, 0}(x), \quad s(0, x)=s_{0}(x) .
$$

We first prove the local existence with the analytic semi-group theory. The local existence and a priori estimates of solutions will allow us to conclude about the global existence.

We introduce the Banach space $X=L^{2}(0, L)^{n+1}$ and the unbounded operator $A$ with the domain $\mathcal{D}(A)$ :

$$
\begin{aligned}
& \mathcal{D}(A)=\left\{u \in X, u^{\prime \prime} \in X, u(0)=0, u^{\prime}(L)=0\right\} \\
& A u=\left(d u_{1}^{\prime \prime}, \ldots, d u_{n}^{\prime \prime}, d_{s} u_{n+1}^{\prime \prime}\right)^{T}
\end{aligned}
$$

where the superscript $T$ denotes the transposition operator. The operator $A$ is an unbounded densely defined operator on $X$. It is the infinitesimal generator of an analytic semi-group on $X$ $[5,11]$. Therefore we obtain the following existence result.

Theorem 3..1. For any functions $\left(c_{i, 0}, s_{0}\right) \in \mathcal{D}\left(A^{\alpha}\right)$ with $\alpha>1 / 2$ such that

$$
0 \leq c_{i, 0} \leq 1, \quad 0 \leq s_{0} \leq 1, \quad i=1, \ldots, n
$$

system (3..1)-(3..4) has a global solution satisfying

$$
\begin{gathered}
\left(c_{1}, \ldots, c_{n}, s\right) \in C^{0}\left([0,+\infty), \mathcal{D}\left(A^{\alpha}\right)\right) \cup C^{1}(] 0,+\infty[, X), \\
0 \leq c_{i} \leq 1, \quad 0 \leq s \leq 1 .
\end{gathered}
$$

Proof. We first re-write system (3..1)-(3..4) as an abstract Cauchy problem. We introduce the mapping $F=\left(F_{1}, \ldots, F_{n+1}\right)^{T}$ defined by

$$
F_{i}(u)=k\left(c_{i-1}-c_{i}\right)-\left(v(u) c_{i}\right)^{\prime}, \text { for } i=1, \ldots, n, \quad F_{n+1}(u)=k_{s} s-(v(u) s)^{\prime},
$$


with

$$
\begin{aligned}
& c_{0}=0, \quad c_{1}=1+u_{1}, \quad c_{i}=u_{i}, i=2, . ., n, \quad u_{n+1}=s . \\
& v(u)(x)=k \int_{0}^{x}\left(c_{1}+\ldots+c_{n}\right)(t) d t+k_{s} \int_{0}^{x} s(t) d t .
\end{aligned}
$$

Then problem (3..1)-(3..4) re-writes as

$$
\frac{d u}{d t}=A u+F(u), \quad u(0)=u_{0} .
$$

We easily check that the nonlinear mapping $F$ is a locally Lipschitz continuous function from $X_{\alpha}$ into $X$ for any $\alpha>1 / 2$. Here we have set $X_{\alpha}=\mathcal{D}\left(A^{\alpha}\right)$. Therefore we conclude that for any $u_{0} \in X_{\alpha}$ problem (3..12) has a unique local solution defined on $[0, T)$.

Here we suppose that $\left(c_{i, 0}, s_{0}\right)$ satisfies (3..7). Let $\left(c_{i}, s\right)$ be the associated local solution on $[0, T)$. Then we set

$$
\begin{aligned}
& a_{0}(t, x)=k\left(c_{1}+\ldots+c_{n}\right)(t, x)+k_{s} s(t, x), \quad b_{0}(x)=k\left(c_{1}+\ldots+c_{n}\right)(t, x), \\
& c_{0}(t, x)=\int_{0}^{x} a_{0}(t, s) d s .
\end{aligned}
$$

Then $\left(C=\left(c_{1}, \ldots, c_{n}\right)^{T}, s\right)$ satisfies the problem $\mathcal{L}(C, s)=0$, with $D=\operatorname{diag}\left(d, . ., d, d_{s}\right) \in$ $\mathcal{M}_{n+1}(\mathbb{R})$,

$$
\begin{gathered}
\mathcal{L}(C, s)=\partial_{t}(C, s)^{T}-D \partial_{x x}^{2}(C, s)^{T}+c_{0}(t, x) \partial_{x}(C, s)^{T}-f(C, s), \\
f(C, s)=\left(-\left(a_{0}(t, x)+k\right) C,-b_{0}(t, x) s+k_{s} s(1-s)\right)^{T}+k\left(0, c_{1}, \ldots, c_{n-1}, 0\right)^{T} .
\end{gathered}
$$

The nonlinear term is a monotone function so that the comparison principle applies for this problem. We introduce the following ordering on $\mathbb{R}^{n+1}$

$$
\forall(a, b) \in \mathbb{R}^{n+1} \times \mathbb{R}^{n+1}, \quad a \leq b \quad \Longleftrightarrow a_{i} \leq b_{i} \forall i=1, \ldots, n+1 .
$$

First, we have $\mathcal{L} 0 \leq \mathcal{L}(C, s)$ with $0 \leq(C, s)^{T}(t, 0)$ and $\partial_{x}(C, s)^{T}(t, L)=0$. Therefore $(C, s)^{T} \geq$ 0 on $[0, T) \times[0, L]$. Next, if we set $e=(1, . ., 1,1) \in \mathbb{R}^{n+1}$, then we obtain that

$$
\mathcal{L} e=a_{0}(t, x)(1, \ldots, 1,0)^{T}+\left(k, 0, \ldots, 0, b_{0}(t, x)\right)^{T} .
$$

Since $(C, s) \geq 0$, then we have from (3..13) that $a_{0}(t, x) \geq 0$ and $b_{0}(t, x) \geq 0$ on $[0, T) \times[0, L]$. Thus $\mathcal{L} e \geq 0$ and $\mathcal{L}(C, s)=0 \leq \mathcal{L} e$ with $e \geq(C, s)^{T}(t, 0)$ and $\partial_{x}(C, s)^{T}(t, L)=\partial_{x} e$. We conclude that

$$
(C, s) \leq e \text { on }[0, T) \times[0, L] .
$$

In order to conclude that $T=+\infty$ it remains to show that $\|(C, s)(t)\|_{\alpha}$ is bounded on $[0, T)$. For that purpose let us note that since the functions $\left(c_{i}, s\right)$ are bounded on $[0, T) \times[0, L]$, the functions $a_{0}, b_{0}$ and $c_{0}$ belong to $L^{\infty}((0, T) \times(0, L))$. Therefore from the standard parabolic estimates we obtain that the function $(C, s)$ belong to $L^{\infty}\left((0, T), W^{2, p}(0, L)\right)$ for any $p \in(1, \infty)$. This estimate prevents the norm $\|(C, s)(t)\|_{\alpha}$ from exploding when $t \rightarrow T^{-}$. We conclude that the solution is global, that is $T=+\infty$. This completes the proof of Theorem 3..1. 


\section{Stability and bifurcation analysis}

\subsection{Disease free equilibrium}

In this section we consider the system corresponding to the normal case, that is with $s=0$. We will study its stationary solutions. They satisfy the following elliptic system of equations:

$$
\begin{gathered}
d c_{i}^{\prime \prime}-\frac{1}{\nu}\left(c_{i} p^{\prime}\right)^{\prime}+k\left(c_{i-1}-c_{i}\right)=0, \quad i=1, \ldots, n, \\
p^{\prime \prime}=\nu k\left(c_{1}+\ldots+c_{n}\right),
\end{gathered}
$$

together with the boundary conditions

$$
\begin{aligned}
& c_{1}(0)=1, \quad c_{i}(0)=0 \quad i=2, \ldots, n, \\
& c_{i}^{\prime}(L)=0, \quad i=1, . ., n, \\
& p^{\prime}(0)=0, \quad p(0)=0 .
\end{aligned}
$$

We will obtain the following result.

Theorem 4..1. System (4..1)-(4..3) has a solution $\left(c_{1,0}, . ., c_{n, 0}, p_{0}\right) \in C^{2}([0, L])^{n+1}$ such that

$$
c_{i} \geq 0, \quad \forall i=1, \ldots, n \text {. }
$$

Proof. The proof of this result uses the Leray-Schauder method. To apply it we introduce the following homotopy, that is the system of equations

$$
\begin{gathered}
d c_{i}^{\prime \prime}-\frac{\tau}{\nu}\left(c_{i} p^{\prime}\right)^{\prime}+k\left(\tau c_{i-1}-c_{i}\right)=0, \quad i=1, \ldots, n, \\
p^{\prime \prime}=\nu k\left|c_{1}+\ldots+c_{n}\right|,
\end{gathered}
$$

together with (4..3). Here $\tau \in[0,1]$ is the homotopy parameter.

Let us consider a solution $\left(c_{1}, \ldots, c_{n}, p\right)$ of problem (4..5), (4..6) and (4..3). From (4..6) we know that $p^{\prime \prime} \geq 0$. We will show that $c_{i} \geq 0$ for any $i=1, \ldots, n$. Indeed the function $c_{1}$ satisfies the equation

$$
d c_{1}^{\prime \prime}-\frac{\tau}{\nu} p^{\prime} c_{1}^{\prime}-\left(\frac{\tau}{\nu} p^{\prime \prime}+k\right) c_{1}=0, \quad c_{1}(0)=1, \quad c_{1}^{\prime}(L)=0 .
$$

Since function $\frac{\tau}{\nu} p^{\prime \prime}+k$ is positive, then $c_{1}(x)$ cannot have negative minima. Hence, it is either nonnegative for all $x \in[0, L]$ or it is nonnegative in some interval $\left[0, x_{0}\right], c_{1}\left(x_{0}\right)=0$, and it is negative in $\left(x_{0}, L\right)$. We note that $c_{1}^{\prime}(x) \neq 0$ for $x_{0}<x<L$. Otherwise it would be a point of maximum, which contradicts the assumption that the function is negative in this interval and $c_{1}\left(x_{0}\right)=0$. Hence, $c_{1}^{\prime}(x)<0$ for $x_{0}<x<L$. Consequently, $c_{1}^{\prime \prime}(L) \geq 0$, and we obtain a contradiction in signs in the equation for $c_{1}$. This contradiction proves that $c_{1}(x)>0$ for $0<x \leq$ $L$.

Next, the function $c_{2}$ satisfies the equation

$$
d c_{2}^{\prime \prime}-\frac{\tau}{\nu} p^{\prime} c_{2}^{\prime}-\left(\frac{\tau}{\nu} p^{\prime \prime}+k\right) c_{2}=-\tau k c_{1} \leq 0, \quad c_{2}(0)=0, \quad c_{2}^{\prime}(L)=0
$$


As above, we conclude that $c_{2} \geq 0$. By induction we show that $c_{i} \geq 0$ for any $i=1, . ., n$. Finally, from the positiveness of the functions $c_{i}$ it follows that the equation for $p$ reduces to $p^{\prime \prime}=\nu k\left(c_{1}+\ldots+c_{n}\right)$.

We obtain a priori estimates of these solutions. Multiplying the equation for $c_{i}$ by $c_{i}$ and integrating over $(0, L)$, we obtain

$$
\begin{gathered}
d \int_{0}^{L}\left(c_{i}^{\prime}\right)^{2} d x+k \int_{0}^{L} c_{i}^{2} d x+d c_{i}^{\prime}(0) c_{i}(0)+\tau k \int_{0}^{L} \frac{c_{i}^{2}}{2}\left(c_{1}+\ldots+c_{n}\right) d x= \\
-\frac{\tau}{\nu} p^{\prime}(L) \frac{c_{i}^{2}(L)}{2}+\tau k \int_{0}^{L} c_{i-1} c_{i} d x .
\end{gathered}
$$

Since $p^{\prime}(L)=\nu k \int_{0}^{L}\left(c_{1}+. .+c_{n}\right)$, then we have $p^{\prime}(L) \geq 0$ and

$$
C|| c_{i} \|_{H^{1}}^{2} \leq-d c_{i}^{\prime}(0) c_{i}(0)+\tau k \int_{0}^{L} c_{i-1} c_{i} d x \leq-d c_{i}^{\prime}(0) c_{i}(0)+k \int_{0}^{L} c_{i-1} c_{i} d x .
$$

Here and below $C$ denotes non-negative constants that do not depend on the solution.

For $i=1$ we obtain:

$$
C\left\|c_{1}\right\|_{H^{1}}^{2} \leq-d c_{1}^{\prime}(0)
$$

while for $i \geq 2$

$$
C\left\|c_{i}\right\|_{H^{1}} \leq k\left\|c_{i-1}\right\|_{L^{2}}
$$

By induction we obtain for $i \geq 2$ :

$$
\left\|c_{i}\right\|_{H^{1}}^{2} \leq C\left\|c_{1}\right\|_{L^{2}}
$$

It remains to estimate $c_{1}^{\prime}(0)$. For that purpose we integrate the equation for $c_{1}$ over $(0, L)$. We obtain:

$$
-d c_{1}^{\prime}(0)=k \int_{0}^{L} c_{1} d x+\frac{\tau}{\nu} p^{\prime}(L) c_{1}(L)
$$

On the one hand, we have $p^{\prime}(L)=\nu k \int_{0}^{L}\left(c_{1}+\ldots+c_{n}\right)$. The Hölder inequality provides that $p^{\prime}(L) \leq C\left(\left\|c_{1}\right\|_{L^{2}}+\ldots+\left\|c_{n}\right\|_{L^{2}}\right)$. Then due to (4..11) we obtain:

$$
0 \leq p^{\prime}(L) \leq C\left\|c_{1}\right\|_{L^{2}}
$$

On the other hand, we consider the equation for $c_{1}$

$$
d c_{1}^{\prime \prime}-\frac{\tau}{\nu} p^{\prime} c_{1}^{\prime}-\left(k+\frac{\tau}{\nu} p^{\prime \prime}\right) c_{1}=0, \quad c_{1}(0)=1, \quad c_{1}^{\prime}(L)=0
$$

Since $p^{\prime \prime} \geq 0$, then from the maximum principle we obtain that $c_{1} \leq 1$. Hence $c_{1}(L) \leq 1$. Therefore, using (4..12), we obtain $-d c_{1}^{\prime}(0) \leq C\left\|c_{1}\right\|_{L^{2}}$. Finally, due to (4..9) we conclude that $\left\|c_{1}\right\|_{H^{1}}^{2} \leq C$ and $\left\|c_{i}\right\|_{H^{1}}^{2} \leq C$ for any $i=1, \ldots, n$. This bound provides an estimate for $p$ in $H^{3}$. Due to the Sobolev embedding theorem we obtain an estimate in $C^{2}([0, L])$ of the function $p$ and due to equation (4..5) we obtain a bound in $C^{1}([0, L])$ for $c_{i}$. 
We now consider the Banach space $Y$ defined by

$$
Y=\left(C^{1}([0, L])\right)^{n} \times C^{2}([0, L])
$$

endowed with the norm

$$
\left\|\left(c_{1}, \ldots, c_{n}, p\right)\right\|_{Y}=\left\|c_{1}\right\|_{C^{1}([0, L])}+\ldots+\left\|c_{n}\right\|_{C^{1}([0, L])}+\|p\|_{C^{2}([0, L])} .
$$

We also consider the mapping $T_{\tau}: Y \rightarrow Y$ defined by $T_{\tau}\left(\left(b_{1}, \ldots, b_{n}\right), q\right)=\left(\left(c_{1}, \ldots, c_{n}\right), p\right)$, where $\left(c_{1}, \ldots, c_{n}, p\right)$ is given by the resolution of the linear system:

$$
\begin{gathered}
d c_{i}^{\prime \prime}-k c_{i}=\frac{\tau}{\nu}\left(\left(b_{i} q^{\prime}\right)^{\prime}-k \tau b_{i-1}\right), \quad i=1, \ldots, n . \\
p^{\prime \prime}=\nu k\left(c_{1}+\ldots+c_{n}\right),
\end{gathered}
$$

together with (4..3). From the elliptic regularity it follows that the mapping $T_{\tau}$ is a compact operator.

Next, from the above a priori estimates it follows that there exists some constant $M>0$ such that for any $\tau \in[0, L],(C, p) \in X$ is a fixed point of $T_{\tau}$ implies that $\|(C, p)\|_{Y}<M$. Therefore if we set $B=B_{Y}(0, M)$, then the topological degree $\operatorname{deg}\left(I-T_{\tau}, B, 0\right)$ is well defined and from the homotopy invariance we have

$$
\operatorname{deg}\left(I-T_{1}, B, 0\right)=\operatorname{deg}\left(I-T_{0}, B, 0\right) .
$$

Moreover, the operator $T_{0}$ corresponds to the constant operator

$$
T_{0}(C, q)=\left(a_{1}^{0}, \ldots, a_{n}^{0}, q^{0}\right)
$$

where $a_{i}^{0} \equiv 0$ for $i \geq 2$, the function $a_{1}^{0}$ is given by the resolution of

$$
d a^{\prime \prime}-k a=0, \quad a(0)=1, \quad a^{\prime}(L)=0,
$$

and $q^{0}(x)=-\int_{x}^{L} \int_{0}^{s} a_{1}^{0}(t) d t d s$. Therefore we obtain $\operatorname{deg}\left(I-T_{0}, B, 0\right)=1$ and, due to (4..18), system (4..5)-(4..6) has a solution. This completes the proof of Theorem 4..1.

We now consider the evolution system

$$
\begin{gathered}
\frac{\partial c_{i}}{\partial t}+\frac{\partial\left(c_{i} v\right)}{\partial x}=d \frac{\partial^{2} c_{i}}{\partial x^{2}}+k\left(c_{i-1}-c_{i}\right), \quad i=1, \ldots, n . \\
\frac{\partial v}{\partial x}=k\left(c_{1}+\ldots+c_{n}\right),
\end{gathered}
$$

together with the boundary conditions

$$
\begin{aligned}
& c_{1}(0)=1, \quad c_{i}(0)=0 \quad i=2, \ldots, n, \\
& c_{i}^{\prime}(L)=0, \quad i=1, . ., n, \quad v(0)=0 .
\end{aligned}
$$


From Theorem $4 . .1$ it follows that this system has a stationary solution $\left(C_{0}, v_{0}\right)$, where $C_{0}=$ $\left(c_{1,0}, \ldots, c_{n, 0}\right)^{T}, v_{0}=\frac{1}{\nu} p_{0}^{\prime}$. Since the evolution problem (4..21)-(4..23) has been formulated as an abstract Cauchy problem, the asymptotic stability of the equilibrium $\left(C_{0}, v_{0}\right)$ can be obtain from the eigenvalue problem: find $\lambda \in \mathbb{C}$ and functions $C=\left(c_{1}, \ldots, c_{n}\right)^{T}, v$ satisfying

$$
\begin{gathered}
d c_{i}^{\prime \prime}+k\left(c_{i-1}-c_{i}\right)-\left(c_{i} v_{0}\right)^{\prime}-\left(c_{i, 0} v\right)^{\prime}=\lambda c_{i}, \\
v^{\prime}=k\left(c_{1}+\ldots+c_{n}\right),
\end{gathered}
$$

with the boundary conditions

$$
c_{i}(0)=0, \quad c_{i}^{\prime}(L)=0, \quad i=1, \ldots, n, \quad v(0)=0 .
$$

We set the operator $A$ defined by:

$$
(A C)_{i}=d c_{i}^{\prime \prime}+k\left(c_{i-1}-c_{i}\right)-\left(c_{i} v_{0}\right)^{\prime}-\left(c_{i, 0} v(C)\right)^{\prime}, \quad \text { with } v(C)(x)=\nu k \int_{0}^{x}\left(c_{1}+\ldots+c_{n}\right)(t) d t .
$$

Our main assumption is that

$$
\sigma_{p}(A) \subset \mathbb{C}^{-},
$$

where $\sigma_{p}(A)$ denotes the spectrum of the operator $A$ and $\mathbb{C}^{-}$is the complex left-half plane. Note that the spectrum of the operator $A$ consists only of the discrete spectrum because it has a compact resolvent. Assumption (4..28) means that the stationary solution of problem (4..21)-(4..23) is asymptotically stable. This corresponds to an exponential convergence of the solution of the evolution problem towards the stationary solution as soon as the initial condition is close to it.

\subsection{Stability analysis and bifurcations}

In this section we study stability of the disease free equilibrium $\left(C_{0}, v_{0}, s_{0}=0\right)$ with respect to the evolution problem (3..1)-(3..4). Since this problem has been formulated as an abstract Cauchy problem, then the asymptotic stability is determined by the location of the spectrum of the linearized problem:

$$
\begin{gathered}
\lambda c_{i}+\left(c_{i, 0} v\right)^{\prime}+\left(c_{i} v_{0}\right)^{\prime}=d c_{i}^{\prime \prime}+k\left(c_{i-1}-c_{i}\right), \quad i=1, \ldots, n, \\
\lambda s+\left(s v_{0}\right)^{\prime}=d_{s} s^{\prime \prime}+k_{s} s, \\
v^{\prime}=k\left(c_{1}+\ldots+c_{n}\right)+k_{s} s
\end{gathered}
$$

together with the boundary conditions

$$
\begin{aligned}
& c_{i}(0)=0 \quad i=1, \ldots, n, \quad s(0)=0, \quad v(0)=0, \\
& c_{i}^{\prime}(L)=0, \quad i=1, \ldots, n, \quad s^{\prime}(L)=0 .
\end{aligned}
$$


We re-formulate this problem as follows: find $\lambda \in \mathbb{C}$ and functions $C=\left(c_{1}, . ., c_{n}\right)^{T}$ and $s$ such that

$$
\begin{aligned}
& \lambda C=A C+M s, \\
& \lambda s=L s,
\end{aligned}
$$

where the operator $A$ is defined in (4..27), and the operators $M$ and $L$ are defined by the equalities

$$
\begin{gathered}
(M s)_{i}=-k_{s}\left(c_{i, 0} \int_{0}^{x} s(t) d t\right)^{\prime}, \\
L s=d_{s} s^{\prime \prime}+k_{s} s-\left(s v_{0}\right)^{\prime} .
\end{gathered}
$$

We define the operator

$$
N(C, s)=\left(\begin{array}{cc}
A & M \\
0 & L
\end{array}\right)\left(\begin{array}{l}
C \\
s
\end{array}\right) .
$$

Therefore, problem (4..33) reads as

$$
N(C, s)=\lambda(C, s)^{T}
$$

Lemma 4..2. The following spectral relations hold:

$$
\sigma_{p}(A) \subset \sigma_{p}(N) \subset \sigma_{p}(A) \cup \sigma_{p}(L) .
$$

Moreover, if assumption (4..28) holds and if $\lambda \in \sigma_{p}(L)$ with $\operatorname{Re} \lambda>0$, then $\lambda \in \sigma_{p}(N)$.

Proof. Let $\lambda \in \sigma_{p}(A)$ be given and $C$ be the corresponding eigenfunction. Then $(C, s=0)$ satisfies $N(C, s)=\lambda(C, s)^{T}$. Hence $\lambda \in \sigma_{p}(N)$.

Next, suppose that $\lambda \in \sigma_{p}(N)$ and $(C, s)$ be the corresponding eigenfunction. If $s \equiv 0$, then $A C=\lambda C$ and $\lambda \in \sigma_{p}(A)$. If $s \neq 0$, then $L s=\lambda s$ and $\lambda \in \sigma_{p}(L)$.

Assume that $\lambda_{0} \in \sigma_{p}(L)$ with $\operatorname{Re} \lambda_{0}>0$ and that $s_{0}$ is the corresponding eigenfunction. From the assumption $\sigma_{p}(A) \in \mathbb{C}^{-}$it follows that the operator $\left(\lambda_{0}-A\right)$ invertible. If we set $C_{0}=$ $\left(\lambda_{0}-A\right)^{-1} M s_{0}$, then

$$
\lambda_{0}\left(C_{0}, s_{0}\right)^{T}=N\left(C_{0}, s_{0}\right),
$$

and $\lambda_{0} \in \sigma_{p}(N)$.

Corollary 4..3. Under assumption (4..28), if $\sigma_{p}(L) \subset \mathbb{C}^{-}$then $\left(C_{0}, v_{0}, s_{0}\right)$ is locally stable. If $\sigma_{p}(L) \cap \mathbb{C}^{+} \neq \emptyset$, then it is unstable.

Consider the eigenvalue problem (4..29)-(4..32). Equation (4..30) can be solved independently of (4..29), (4..31). It represents itself an eigenvalue problem for the operator

$$
L s=d_{s} s^{\prime \prime}-\left(v_{0} s\right)^{\prime}+k_{s} s
$$


Proposition 4..4. The principal eigenvalue of the operator $L$ is an increasing real valued function of $k_{s}$. There exists $k_{s, 0}$ such that it is positive for $k_{s}>k_{s, 0}$ and negative for $k_{s}<k_{s, 0}$. The principal eigenvalue is negative for $d_{s}$ sufficiently large.

Proof. From the properties of scalar second order operators, it follows that the principal eigenvalue of the operator $L$ is real. Note that eigenvalues $\lambda\left(d_{s}, k_{s}\right)$ of the operator $L$ can be written as

$$
\lambda\left(d_{s}, k_{s}\right)=\lambda\left(d_{s}\right)+k_{s},
$$

where $\lambda\left(d_{s}\right)$ is the corresponding eigenvalue of the operator

$$
L_{1} s=d_{s} s^{\prime \prime}-\left(v_{0} s\right)^{\prime} .
$$

From this follows the first assertion of the proposition on the dependence of the principal eigenvalue on $k_{s}$.

Next, let us study its dependence on $d_{s}$. Since $L$ is a scalar second order operator, we can use the method of test functions: if there exists $u$ such that it satisfies the boundary conditions and $L u<0$ for all $x$, then all eigenvalues are in the left-half plane.

Let us take the test function

$$
u(x)=a x(2 L-x) .
$$

We note that $v_{0}(x)$ is an increasing function, therefore $v_{0} u$ is also increasing on the interval $(0, L)$, its derivative is positive. Therefore we obtain $L u=-2 a d_{s}-\left(v_{0} u\right)^{\prime}+k u<0$ for $d_{s}$ sufficiently large. The proposition is proved.

The results of this section allow us to study bifurcations of solutions of the problem

$$
\begin{gathered}
d \frac{d^{2} c_{i}}{d x^{2}}-\frac{d\left(c_{i} v\right)}{d x}+k\left(c_{i-1}-c_{i}\right)=0, \quad i=1, \ldots, n, \\
d_{s} \frac{d^{2} s}{d x^{2}}-\frac{d(s v)}{d x}+k_{s} s=0, \\
\frac{d v}{d x}=k\left(c_{1}+\ldots+c_{n}\right)+k_{s} s, \\
c_{1}(0)=1, \quad c_{i}(0)=0 \quad i=2, \ldots, n, \quad s(0)=0, \\
\left.\frac{d c_{i}}{d x}\right|_{L}=0, \quad i=1, \ldots, n,\left.\quad \frac{d s}{d x}\right|_{L}=0, \quad v(0)=0 .
\end{gathered}
$$

We will suppose that $d_{s}$ and $k_{s}$ depend on a parameter $\tau$ and recall that this problem has a disease free solution $C=C_{0}, v=v_{0}, s=0$ independent of $\tau$.

Theorem 4..5. Suppose that the principal eigenvalue $\lambda_{0}(\tau)$ of the operator $L$ given by (4..38) crosses the origin for some $\tau=\tau_{0}$, it is negative for $\tau<\tau_{0}$ and positive for $\tau>\tau_{0}$. Then the disease free solution is unstable for $\tau>\tau_{0}$ with respect to problem (3..1)-(3..4). Instead of it, there is another stable stationary solution with $s \neq 0$. 
Proof. Let us consider the Banach manifold

$$
\begin{gathered}
\mathcal{M}=\left\{\left(c_{1}, \ldots, c_{n}, s\right) \in C^{2}([0, L])^{n+1}, c_{1}(0)=1, c_{i}(0)=0, i=2, \ldots, n,\right. \\
\left.c_{i}^{\prime}(L)=0, i=1, \ldots, n, s(0)=0, s^{\prime}(0)=0\right\}
\end{gathered}
$$

and the operator $R_{\tau}$ acting from $\mathcal{M}$ into the Banach space $Z=C^{0}([0, L])^{n+1}$ defined by the equality $R_{\tau}\left(c_{1}, . ., c_{n}, s\right)=\left(f_{1}, . ., f_{n}, g\right)$ where

$$
\begin{gathered}
d c_{i}^{\prime \prime}-\left(c_{i} v(c, s)\right)^{\prime}+k\left(c_{i-1}-c_{i}\right)=f_{i}, \quad i=1, \ldots, n, \\
d_{s} s^{\prime \prime}+(s v(c, s))^{\prime}+k_{s} s=g
\end{gathered}
$$

and

$$
v(c, s)(x)=\int_{0}^{x}\left(k\left(c_{1}+\ldots+c_{n}\right)+k_{s} s\right) d t .
$$

The disease free stationary solution $C=C_{0} \equiv\left(c_{1,0}, \ldots, c_{n, 0}\right), v=v_{0}, s=0$ provided by Theorem $4 . .1$ satisfies the equation

$$
R_{\tau}\left(C_{0}, 0\right)=0
$$

for any $\tau$. It can be verified that the linearized operator $R_{\tau}^{\prime}$ is a Fredholm operator with the zero index. The nonlinear operator is proper on bounded closed sets. Therefore, we can define the topological degree.

The index of the solution $\left(C_{0}, 0\right)$ equals, by definition, the topological degree over a small sphere $B_{\varepsilon}\left(C_{0}, 0\right)$ in the function space containing the solution:

$$
\operatorname{index}\left(R_{\tau}\left(C_{0}, 0\right)\right)=\lim _{\varepsilon \rightarrow 0} \operatorname{deg}\left(R_{\tau}, B_{\varepsilon}\left(C_{0}, 0\right)\right),
$$

with $B_{\varepsilon}=\left\{(c, s) \in \mathcal{M}, \quad\left\|(c, s)-\left(C_{0}, 0\right)\right\|_{C^{2}}<\varepsilon\right\}$. On the other hand, the index can be computed by the following formula

$$
\operatorname{index}\left(R_{\tau}\left(C_{0}, 0\right)\right)=(-1)^{\nu\left(k_{s}\right)},
$$

where $\nu\left(k_{s}\right)$ is the sum of the multiplicities of positive eigenvalues of the operator $R_{\tau}^{\prime}\left(C_{0}, 0\right)$ linearized about the solution $\left(C_{0}, 0\right)$.

From the definition of the operator $N$ (see (4..36)) it follows that

$$
\left(R_{\tau}^{\prime}\left(C_{0}, 0\right)\right)(C, s)=N(C, s)^{T} .
$$

Therefore, if all eigenvalues of the operator $N$ are in the left-half plane, then the index of the solution $\left(C_{0}, 0\right)$ equals 1 . If a simple real eigenvalue crossed the origin, then the index changes to -1 . Since the topological degree does not change under a small deformation of the operator, and it equals the sum of indices of the solutions, then there is another solution that appears for $\tau>\tau_{0}$. We note that there are no other disease free solutions in a small neighborhood of $\left(C_{0}, 0\right)$. Hence, $s \neq 0$ for the bifurcating solution. 


\section{Numerical simulations}

This section is devoted to numerical simulations of problem (3..1)-(3..4). We use a semi-implicit finite difference scheme for equations (3..1) and (3..2) while equation (3..3) is solved by using an implicit Euler scheme. We take the following values of the parameters

$$
n=3, \quad L=1, \quad d=0.1, \quad k=15 .
$$

We first consider the normal case, that is $s \equiv 0$. For the values of parameters $(5 . .1)$ the evolution system converges towards a stationary state. It is given in Fig.1. The convergence is very fast (about 400 iterations with a time step $\delta=0.01$ ). This observation corresponds to the assumption (4..28) about the location of the point spectrum.
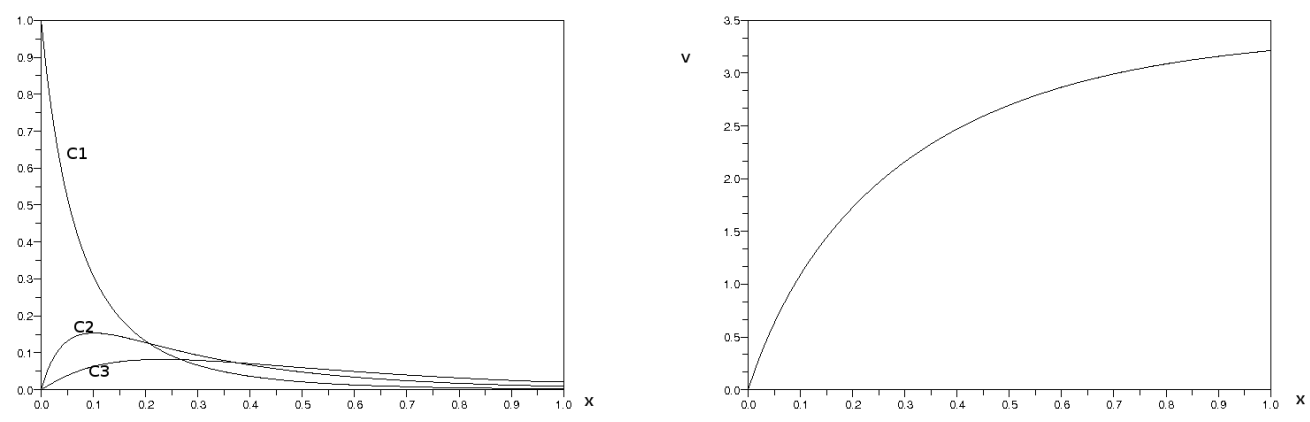

Fig. 1: Disease free equilibrium. The concentrations of immature cells $c_{1}, c_{2}$ and $c_{3}$ on the left and the velocity of cell motion on the right.

Figure 2 (left) shows the principal eigenvalue of the operator $L_{1}$ (see (4..40)) as a function of $d_{s}$. There are two curves, one of them obtained for the space step $\delta x=5.10^{-2}$, another one with the step two times less. The two curves coincide. The stability boundary for the disease free stationary solution is shown in Figure 2 (right). The stability region is below the curve, and the instability region above it.
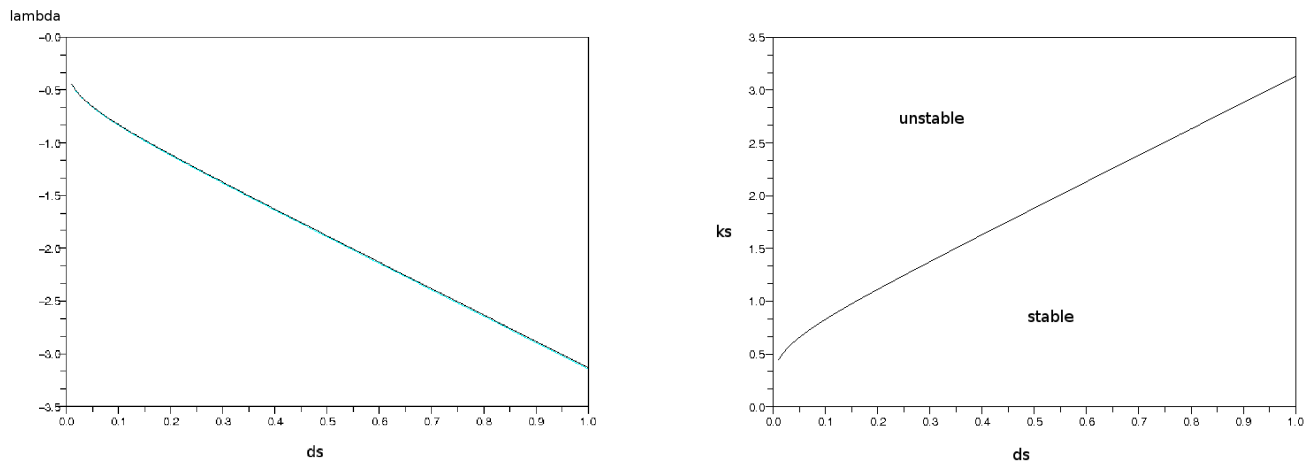
Fig. 2: Principal eigenvalue $\lambda\left(d_{s}\right)$ of the operator $L_{1}$ as a function of $d_{s}$ (left). Stability curve of the disease free equilibrium (corresponding to (5..1)) (right).

In order to check the validity of the stability analysis we consider system (3..1)-(3..4) with a small initial concentration of leukemic cells and for different values of parameters $k_{s}$ and $d_{s}$. The perturbation tends to zeros as time increases if $k_{s}$ is sufficiently small (Figure 3 (left)). It grows if $k_{s}$ is sufficiently large (Figure 3 (right)). In this case a new stable stationary solution appears. It is shown in Figure 4. Note that for $d_{s}=0.8$, the disease free stationary solution is stable for $k_{s} \leq 2.5$ and unstable for $k_{s} \geq 2.8$. This result is in good agreement with the stability curve in Figure 2 .
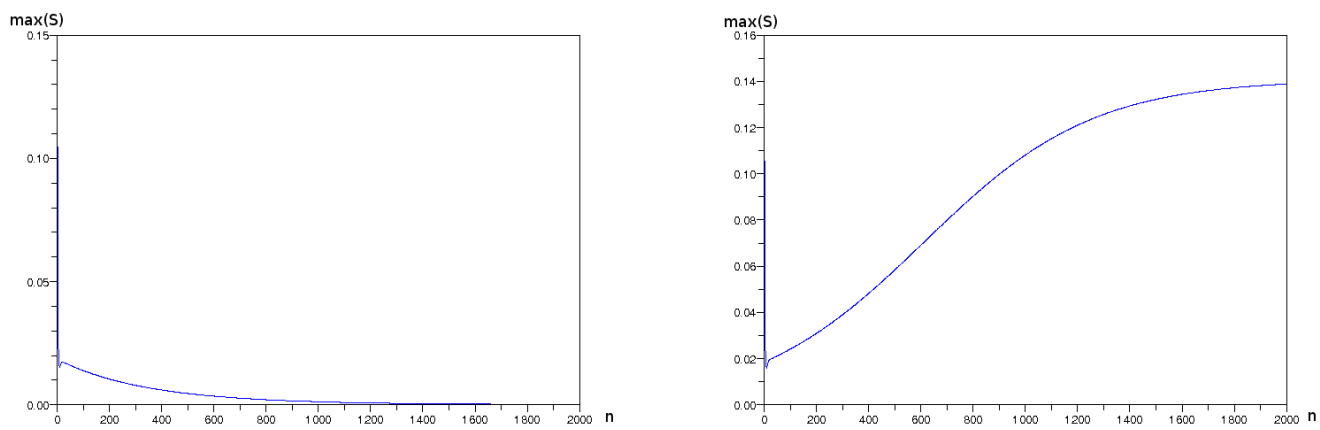

Fig. 3: Evolution of the $\max _{x} s(x, t)$ in time,

$d_{s}=0.8, k_{s}=2.5$ on the left and $k_{s}=2.8$ on the right.
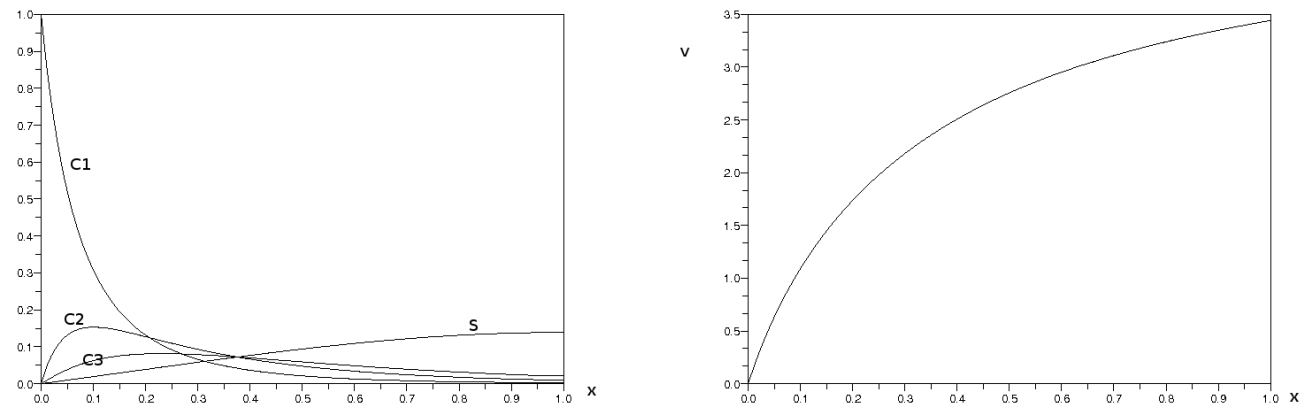

Fig. 4: Leukemic stationary solution, $d_{s}=0.8$ and $k_{s}=2.8$.

Next, we study the dependence of the stability boundary on parameters $d$ and $k$. It strongly depends on $d$ (Figure 5 (left)) while its dependence on $k$ is weak. The solution becomes more stable when $d$ increases. 

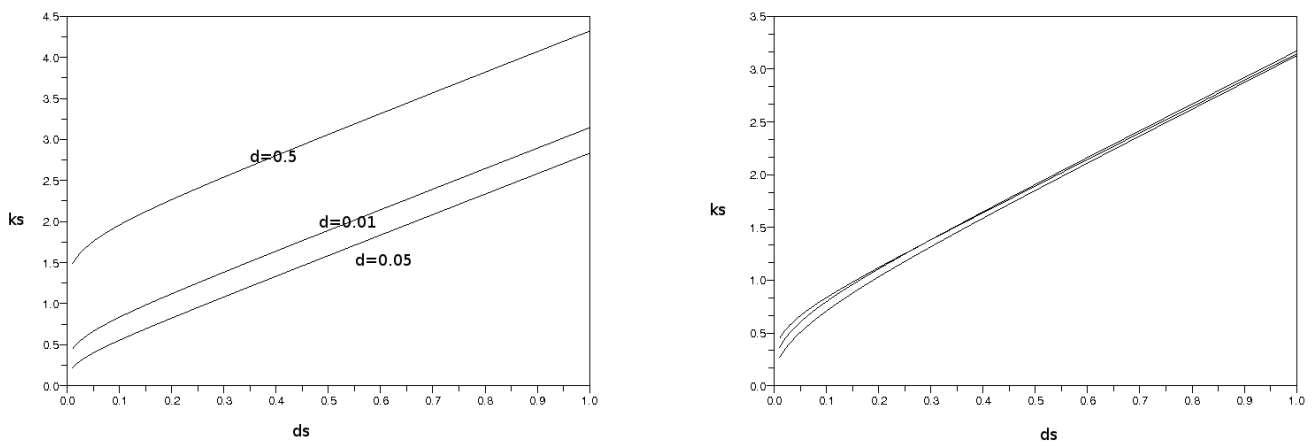

Fig. 5: Stability boundary on the $\left(d_{s}, k_{s}\right)$-plane: $k=15$ and $d=0.05,0.1,0.5$ ( left); $d=0.1$ and $k=15,30,50$ ( right).

Figure 6 shows the influence of the parameter $k_{s}$ on the leukemic stationary solution. The function $s(x)$ has the same qualitative behavior for different values of $k_{s}$ and increases with the increase of this parameter. The convergence to the stationary solution is also faster for larger $k_{s}$.

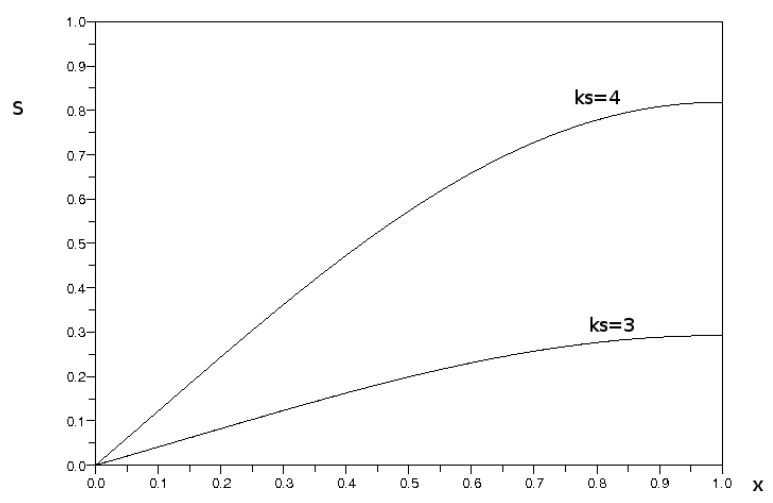

Fig. 6: Leukemic stationary solution for $k_{s}=3$ and $k_{s}=4\left(d_{s}=0.8\right)$.

In order to study the influence of medical treatment on leukemia development we suppose that it acts on the proliferation rates $k$ and $k_{s}$ both for normal and leukemic cells. The treatment is supposed to be periodic in time. The functions $k(t)$ and $k_{s}(t)$ are considered in the form

$$
k(t)=k-a(1+\sin \omega t), \quad k_{s}(t)=k_{s}-a(1+\sin \omega t) .
$$

The results of the computation are shown in Figure 7. 


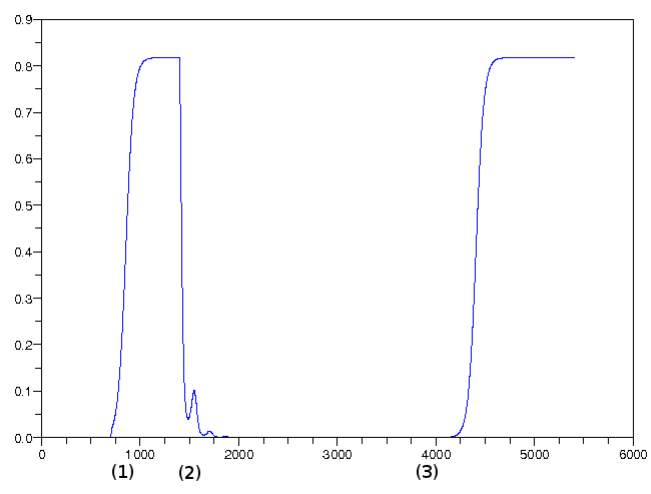

Fig. 7: Concentration of leukemic cells at the boundary between the bone marrow and blood vessels as a function of time.

Time (1) corresponds to the introduction of leukemic cells in the bone marrow. The concentration of malignant cells rapidly increases. Time (2) is the beginning of the treatment. The number of malignant cells drastically decreases because of the decreasing of their proliferation rate. We stop the treatment at time (3). After some times the number of leukemic cells increases and comes back to its original value. The parameters for the numerical simulations are taken as follows: $k=15$, $k_{s}=4, d=0.1, d_{s}=0.8, a=\frac{k_{s}}{2}$, and $\omega=1$.

\section{Discussion}

We consider in this work a simplified hematopoiesis where stem cells give only two cell lineages $A$ and $B$. Cells of the first lineage have several consecutive divisions. In leukemic hematopoiesis all cells are similar to each other. When a cell divides, it gives two daughter cells identical to the mother cell.

It is known that leukemia develops from a single malignant cell. Normal and leukemic hematopoiesis coexist in the bone marrow. They are parallel and do not interact except for the competition for space. Cells push each other out of the bone marrow to blood vessels. Since leukemic cells proliferate faster than normal cells, they can finally win the competition and replace most of normal cells. Moreover, they create an excessive pressure and, consequently, a higher speed of motion. Therefore, normal cells that remain in the bone marrow do not have enough time to become mature.

We have observed this behavior in the individually based modelling [4]. The results of this work and of [3] confirm the conclusion about the excessive pressure and faster cell motion.

The results of this work can be interpreted as follows. Blood cell production in the bone marrow can be described with a reaction-diffusion system and convection in a porous medium. This evolution problem has a stationary solution corresponding to the normal (disease free) case. If $k_{s}$ is sufficiently large or $d_{s}$ sufficiently small, then this solution becomes unstable. Instead, there is another stable stationary solution which appears. It corresponds to the leukemic case. 
Small diffusion coefficient can be related to a larger adhesion of leukemic stem cells to the porous matrix. Large $k_{s}$ corresponds to a faster proliferation of leukemic cells.

The results of this work allow us to make some prediction about the influence of chemotherapy on leukemia development. It is known that medical treatment acts through proliferation of cells, both normal and leukemic. We can take this into account decreasing the parameters $k$ and $k_{s}$. This means that more cells die due apoptosis and less cells proliferate. The results of the numerical simulations show that in the framework of the model considered here leukemia development is not sensitive to $k$ but is strongly influenced by $k_{s}$. Therefore, we can expect that treatment can stop or weaken leukemia. This hypothesis is confirmed by the results of numerical simulations.

However, the concentration of leukemic cells increases when the treatment is stopped. This phenomenon has been clinically observed. The reason for this is that there are some leukemic cells remaining in the bone marrow during the treatment. Their number decreases and probably becomes very small if the treatment is sufficiently long but in the model they never completely disappear. When the treatment is stopped, the proliferation rate of leukemic cells comes back to its initial value. After some time they fill again the bone marrow. These conclusions are in agreement with the medical data [10].

We note finally that in order to model leukemia development in the bone marrow it is necessary to take into account spatial distributions of cells and their motion.

\section{References}

[1] M. Adimy, L. Pujot-Menjouet. A mathematical model describing cellular division with a proliferating phase duration depending on the maturity of cells. EJDE., 107 (2003), 1-14.

[2] D. Ambrosi, F. Mollica. Mechanical Models in Tumor Growth, 122-143, Eds. Preziosi, Chapman and Hall, New-York, 2003.

[3] N. Bessonov, A. Ducrot, V. Volpert. Modelling of Leukemia development in the bone marrow. Proc. of the annual Symposium on "Mathematics applied in Biology and Biophysics", pp. 79-88, 2005.

[4] N. Bessonov, L. Pujo-Menjouet, V. Volpert, Cell modelling of hematopoiesis. Math. Model. Nat. Phenom., 1 (2006), No. 2, 81-103.

[5] D. Henry. Geometric theory of semilinear parabolic equations. Springer-Verlag, Berlin, 1981.

[6] M.C. Mackey. Unified hypothesis of the origin of aplastic anemia and periodic hematopoiesis. Blood, 51 (1978), 941-956.

[7] M.C. Mackey. Dynamic hematological disorders of stem cell origin, Biophysical and biochemical information transfer in recognition. 373-409, Eds. Vassileva-Popova J.G., Jensen E.V. Plenum Press, New-York, 1979. 
[8] M.C. Mackey, R. Rudnicki. Global stability in a delayed partial differential equation describing cellular replication. J. Math. Biol., 33 (1994), 89-109.

[9] M.C. Mackey and R. Rudnicki, A new criterion for the global stability of simultaneous cell replication and maturation process, J. Math. Biol., 38, 1999, 69-76.

[10] F. Michor, T.P. Hughes, Y. Iwasa, S. Branford, N.P. Shah, C.L. Sawyers, M.A. Nowak. Dynamics of chronic myeloid leukemia. Nature, Letters, 435, 2005, 1267-1270.

[11] A. Pazy. Semigroup of linear operators and applications to partial differential equations. Springer-Verlag, New York, 1983.

[12] L. Pujo-Menjouet, R. Rudnicki. Global stability of cellular populations with unequal division. Can. Applied Math. Quart., 8 (2000), 185-203.

[13] E. Trucco. Mathematical models for cellular systems: The Von Foerster equation. Part I. Bull. Math. Biophys., 27 (1965), 285-304.

[14] E. Trucco. Mathematical models for cellular systems: The Von Foerster equation. Part II. Bull. Math. Biophys., 27 (1965), 449-470.

[15] H. Von Foerster. Some remarks on changing populations. The kinetics of cellular proliferation (F. Stohlman eds.), Grune and Stratton, New-York, 382-407, 1959. 